\section{Mamma-Ca.: Verbessern Statine das Überleben?}

\begin{abstract}
Eine antineoplastische Wirkung von Statinen konnte in präklinischen Studien mit Brustkrebszellen bereits nachgewiesen werden. Eine Metaanalyse untersuchte nun den möglichen Zusammenhang zwischen Statineinnahme und Outcome von Brustkrebspatientinnen.
\end{abstract}

\begin{abstract}
tatine werden häufig zur Behandlung der Hyperlipidämie und kardiovaskulärer Erkrankungen eingesetzt. Die Wirkung beruht auf einer Senkung des Cholesterinspiegels im Blut durch die Hemmung der 3-Hydroxy-3-MethylglutarylCoenzym-A-Reduktase (HMG-CoA). Es zeigte sich, dass Statine auch antiapoptotische, antiinvasive und antiproliferative Effekte aufweisen. Metaanalysen belegten, dass die Statineinnahme mit einer verbesserten Überlebensrate bei kolorektalen Tumoren und dem Prostatakarzinom assoziiert ist. Untersuchungen ergaben auch, dass lipophile Statine einen direkt hemmenden Effekt auf Brustkrebszellen in vitro sowie im murinen Mammakarzinommodell aufweisen. Aus ersten klinischen Beobachtungen lässt sich folgern, dass Sta-
\end{abstract}

tine bei Mammakarzinomen, die hohe Konzentrationen von HMG-CoA exprimieren, positive therapeutische Effekte haben. Die Expression von HMG-CoA in Brustkrebszellen scheint somit eine prognostische Bedeutung zu haben. Für die aktuelle Metaanalyse erwiesen sich von 817 Abstracts 14 Studien für die Frage des Effekts von Statinen auf die Überlebenszeit beim Mammakarzinom geeignet. 75.684 Frauen wurden einbezogen.

Unter Statingabe ergab sich für die $\mathrm{Pa}$ tientinnen ein verbessertes rezidivfreies Überleben. Die detaillierte Analyse zeigte, dass der positive Effekt ausschließlich bei lipophilen und nicht bei hydrophilen Statinen auftrat. Auch fand sich eine verbesserte Gesamtüberlebenszeit unter Statineinnahme. Es gibt zudem Hinwei- se, dass Statine das krebsspezifische Überleben verbessern.

Manthravadi S et al. Impact of statins use on cancer recurrence and mortality in breast cancer: a systematic review and meta-analysis. Int J Cancer 2016; 139:1281-8

\section{Kommentar}

Diese Metanalyse reiht sich in eine Serie von Studien zu positiven Nebeneffekten der Statine ein. Da HMG-CoA von Brustkrebszellen exprimiert wird, ist die theoretische Wirkweise der Statine auf die Rezidivfreiheit beim Mammakarzinom im Ansatz erklärbar. Zu betonen ist, dass sich diese positiven Effekte nur bei lipophilen Statinen fanden. Die genaue Bedeutung von Statinen beim Mammakarzinom sollten prospektive Studien klären.

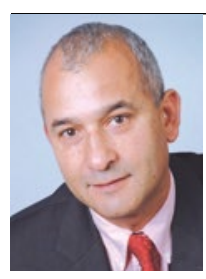

Prof. Tino F. Schwarz Zentrallabor, Stiftung Juliusspital, Würzburg

ful waiting" und das Risiko für neonatale Todesfälle bis zur 37. SSW die Waage hielten. Wurde die Entbindung jedoch um eine Woche bis zur 38. SSW verzögert, erhöhte sich die Mortalität um 8,8/1.000 aufgrund vermehrt auftretender Totgeburten.

Um das Risiko für perinatalen Tod zu minimieren, solle daher in dieser Gruppe mit unkompliziertem Schwangerschaftsverlauf die Entbindung nicht über die 37. SSW hinausgeschoben werden. Bei monochorialen Schwangerschaften scheint das Risiko für Totgeburten nach der 36. SSW höher zu sein als für neonatalen Tod.

Allerdings konnten die Autoren anhand der Daten keine definitive Aussage für diese Zwillingsgruppe machen. Es gebe keine verlässliche Evidenz, die Routineentbindung vor der 36. SSW zu empfehlen.

Peter Leiner

Cheong-See $\mathrm{F}$ et al. Prospective risk of stillbirth and neonatal complications in twin pregnancies: systematic review and meta-analysis. BMJ 2016; 354: i4353 